\title{
ON HEREDITARY PROPERTIES OF EXTREMALLY DISCONNECTED FRAMES AND NORMAL FRAMES
}

\author{
JAVIER GUTIÉRREZ GARCÍA, TOMASZ KUBIAK, AND JORGE PICADO
}

Dedicated to Aleš Pultr on the occasion of his eightieth birthday

\begin{abstract}
Aвstract. Hereditary extremal disconnectedness of frames and its equivalent form of complete extremal disconnectedness are the topic of this paper. We study them in parallel with the corresponding normality properties of frames. Among several characterizations, we show that a frame is hereditarily normal [resp., hereditarily extremally disconnected] if and only if all its open and dense sublocales [resp., closed and dense sublocales] are normal [resp., extremally disconnected]. Even if spaces typically have more sublocales than subspaces, hereditary normality and hereditary extremal disconnectedness are shown to be conservative extensions of the classical properties. Furthermore, we provide such a general setting that permits us to treat several variants of the concepts under study in a unified way. Some of the presented results are new for the traditional topological spaces.
\end{abstract}

\section{INTRODUCTION AND INITIAL OBSERVATIONS}

The purpose of this paper is to study hereditary extremal disconnectedness of frames and its equivalent form of complete extremal disconnectedness vis-à-vis to hereditary normality.

There is a specific duality between topological normality $(\mathrm{N})$ and extremal disconnectedness (ED) that relies on the circumstance that a space is $\mathrm{N}$ [resp., ED] if and only if every two disjoint closed [resp., open] sets are separated by disjoint open [resp., closed] sets. We refer to [22, 23, 24], and especially to the recent paper [17], for more details. A similar duality holds in a pointfree context (cf. Definition 1.4).

Both $\mathrm{N}$ and ED fail to be hereditary properties. They are, respectively, closed-hereditary and open-hereditary - i.e. each closed [resp., open] subspace of a normal space [resp., extremally disconnected space] is normal [resp., extremally disconnected]. The topological cases are well-known. For the pointfree cases we refer to [17] and [15]. It may be worth mentioning that hereditary ED is useful in modal logic (cf. [3]).

Date: January 28, 2019.

2010 Mathematics Subject Classification. 06D22; 54D15.

Key words and phrases. Frame, locale, sublocale, normality, extremal disconnectedness, hereditary normality, complete normality, hereditary extremal disconnectedness, complete extremal disconnectedness, density. 
It is well known that a topological space $X$ is hereditarily normal $(\mathrm{HN})$ if and only if it is completely normal $(\mathrm{CN})$ - i.e. if every two separated subsets of $X$ are separated by disjoint open subsets of $X$ (recall that $A, B \subseteq X$ are separated if $\bar{A} \cap B=\varnothing=A \cap \bar{B})$.

Bearing in mind the duality between $\mathrm{N}$ and ED, one can consider the concept of a completely extremally disconnected (CED) space as one in which every two separated subsets are separated by disjoint closed subsets. This is of course equivalent to the statement that every two separated sets have disjoint closures. Without being named, such a concept was investigated in [4] (also cf. [7]) and proved to be equivalent to hereditary ED. We record the following:

Proposition 1.1. ([4]). The following are equivalent for any topological space X:

(1) $X$ is hereditarily extremally disconnected.

(2) Any two separated subsets of $X$ have disjoint closures.

(3) $X$ is completely extremally disconnected.

A characterization of $\mathrm{CN}$ solely in terms of the lattice $\Omega(X)$ of all open sets of $X$ is given in [30]:

Proposition 1.2. ([30,11]) The following are equivalent for any topological space $X:$

(1) $X$ is completely normal.

(2) For every open sets $A, B \subseteq X$, there exist open sets $U, V \subseteq X$ such that $U \cap V=\varnothing, A \cup U \supseteq B$ and $B \cup V \supseteq A$.

When phrased in terms of the dual lattice $\Omega(X)^{o p}$, condition (2) of Proposition 1.2 becomes a characterization of $C E D$. Indeed, the following holds:

Proposition 1.3. The following are equivalent for any topological space X:

(1) $X$ is completely extremally disconnected.

(2) For any open sets $A, B \subseteq X, \overline{A-B} \cap \overline{B-A}=\varnothing$.

(3) For any open sets $A, B \subseteq X$, there exist open sets $U, V \subseteq X$ such that $U \cup V=$ $X, A \cap U \subseteq B$ and $B \cap V \subseteq A$.

Proof. (1) $\Rightarrow(2)$ : Let $A$ and $B$ be open in $X$. The result follows immediately from Proposition 1.1 and the fact that $A-B$ and $B-A$ are separated. Indeed, $(A-B) \cap \overline{B-A} \subseteq A \cap \overline{X-A}=\varnothing$ and dually.

$(2) \Rightarrow(3)$ : Let $A$ and $B$ be open in $X$ and take $U=X-\overline{A-B}$ and $V=$ $X-\overline{B-A}$. By hypothesis $\overline{A-B} \cap \overline{B-A}=\varnothing$ and thus $U \cup V=X$. On the other hand, $A-B \subseteq \overline{A-B}=X-U$ and so $A \cap U \subseteq B$. Dually, we have that $B \cap V \subseteq A$.

$(3) \Rightarrow(1)$ : We prove that $X$ satisfies condition (2) in Proposition 1.1. Let $A, B \subseteq X$ be separated. Consider $U=X-\bar{A}$ and $V=X-\bar{B}$. By hypothesis there exist open sets $U^{\prime}, V^{\prime} \subseteq X$ such that $U^{\prime} \cup V^{\prime}=X, U \cap U^{\prime} \subseteq V$ and $V \cap V^{\prime} \subseteq U$. Since $B \subseteq X-\bar{A}=U$ and $B \subseteq X-V$ it follows that

$$
B \subseteq U-V \subseteq U-\left(U \cap U^{\prime}\right)=U-U^{\prime} \subseteq X-U^{\prime} .
$$


Dually, $\bar{A} \subseteq X-V^{\prime}$. Hence $\bar{A} \cap \bar{B} \subseteq X-\left(U^{\prime} \cup V^{\prime}\right)=\varnothing$.

Following [33], a topological property $P$ is said to be lattice-invariant if, when $X$ has $P$ and the lattices $\Omega(X)$ and $\Omega(Y)$ are isomorphic, the space $Y$ has property $P$ too. In particular, Propositions 1.2 and 1.3 show that both complete $\mathrm{N}$ and complete ED are lattice-invariant.

Moreover, Propositions 1.2 and 1.3 also indicate how to formulate complete $\mathrm{N}$ and complete ED in an arbitrary (bounded) lattice. We thus have the following definitions:

Definitions 1.4. Let $L$ be a (bounded) lattice with bounds 0 and 1 . Then:

(1) $L$ is normal if

$$
\forall a, b \in L[a \vee b=1 \Rightarrow \exists u, v \in L: u \wedge v=0, a \vee u=1=b \vee v]
$$

(2) $L$ is extremally disconnected if the dual lattice $L^{o p}$ is normal -i.e.

$$
\forall a, b \in L[a \wedge b=0 \Rightarrow \exists u, v \in L: u \vee v=1, a \wedge u=0=b \wedge v] .
$$

(3) $L$ is completely normal if

$$
\forall a, b \in L \quad \exists u, v \in L: u \wedge v=0, a \vee u \geq b, b \vee v \geq a .
$$

(4) $L$ is completely extremally disconnected if $L^{o p}$ is completely normal - i.e.

$$
\forall a, b \in L \exists u, v \in L: u \vee v=1, a \wedge u \leq b, b \wedge v \leq a \text {. }
$$

Remark 1.5. Condition $(\mathrm{CN})$ appears in $[2,2.4]$ under the name of relative normality). Note that (CN) is really stronger than (N), for if $a \vee b=1$, then $a \vee u \geq b$ and $b \vee v \geq a$ imply $a \vee u=b \vee v=1$. By a dual argument, (CED) implies (ED).

Fact 1.6. Any completely normal and extremally disconnected lattice is completely extremally disconnected. Dually, any normal and completely extremally disconnected lattice is completely normal.

Proof. Let $a, b \in L$. By complete normality there exist $u, v \in L$ such that $u \wedge v=0, a \vee u \geq b$ and $b \vee v \geq a$. Since $L$ is extremally disconnected there exist $x, y \in L$ such that $x \vee y=1, x \wedge v=0$ and $y \wedge u=0$. Hence $a \wedge x \leq(b \vee v) \wedge x \leq b$ and $b \wedge y \leq(a \vee u) \wedge y \leq a$.

Fact 1.7. The following are equivalent for any lattice $L$ :

(1) $L$ is completely normal and extremally disconnected.

(2) L is normal and completely extremally disconnected.

(3) L is completely normal and completely extremally disconnected.

In particular, if $L=\Omega(X)$, then $X$ is HN and ED if and only if $X$ is $N$ and HED (cf. [13, 6R] and [25]) if and only if $X$ is HN and HED. 


\section{SOME BACKGROUND ON POINTFREE TOPOLOGY}

We recall some basic notions and facts about frames and locales. For further information see [28].

The category Frm of frames has as objects those complete lattices $L$ in which

$$
a \wedge \bigvee B=\bigvee\{a \wedge b \mid b \in B\}
$$

for all $a \in L$ and $B \subseteq L$. Morphisms, called frame homomorphisms, are those maps between frames that preserve arbitrary joins (in particular, the bottom element 0 ) and finite meets (in particular, the top element 1 ).

The lattice $\Omega(X)$ of open subsets of a space $X$ is the typical example of a frame. For any continuous map $f: X \rightarrow Y$, the mapping $\Omega(f): \Omega(Y) \rightarrow \Omega(X)$ defined by $\Omega(f)(U)=f^{-1}[U]$ is a frame homomorphism. The category of locales is the opposite category of Frm.

Remark 2.1. Note that in the case of a frame $L$, conditions (CN) and (CED) are, by Propositions 1.2 and 1.3, both conservative extensions of their topological counterparts - i.e. a topological space $X$ is $C N$ (resp., ED) if and only if the frame $L=\Omega(X)$ satisfies $C N$ (resp., CED).

With $L$ a frame and $a \in L$, the map $a \wedge(\cdot): L \rightarrow L$ preserves arbitrary joins and thus has a right (Galois) adjoint $a \rightarrow(\cdot): L \rightarrow L$ determined by

$$
a \wedge c \leq b \text { iff } c \leq a \rightarrow b .
$$

Thus, $a \rightarrow b=\bigvee\{c \in L \mid a \wedge c \leq b\}$. The pseudocomplement of $a \in L$ is $a^{*}=a \rightarrow 0$. Then: $a \wedge a^{*}=0, a \leq a^{* *}, a^{* * *}=a^{*}$ and $(\bigvee A)^{*}=\bigwedge_{a \in A} a^{*}$ for all $A \subseteq L$ (but the dual de Morgan law is not true in general; we only have $\left.(\bigwedge A)^{*} \geq \bigvee_{a \in A} a^{*}\right)$. In particular, $(\cdot)^{*}$ is order-reversing.

An $S \subseteq L$ is a sublocale of $L$ if, for any $A \subseteq S, a \in L$ and $b \in S$, we have $\bigwedge A \in S$ (in particular, $1 \in S$ ) and $a \rightarrow b \in S$. The set $\mathcal{S}(L)$ of all sublocales of $L$ forms a coframe (i.e. a complete lattice satisfying the dual of (2.1)) under inclusion. Arbitrary infima coincide with intersections, $\{1\}$ is the bottom element and $L$ is the top element. Regarding suprema, there is the formula

$$
\bigvee_{i \in I} S_{i}=\left\{\bigwedge A \mid A \subseteq \bigcup_{i \in I} S_{i}\right\}
$$

for every $\left\{S_{i} \mid i \in I\right\} \subseteq \mathcal{S}(L)$.

Since $\mathcal{S}(L)$ is the dual of a complete Heyting algebra, it has co-pseudocomplements, usually called pseudodifferences or remainders (see [12] for more information). We shall denote the pseudodifference of an $S$ in $\mathcal{S}(L)$ by $L \backslash S$ and will use the formula

$$
L \backslash S=\bigcap\{R \in \mathcal{S}(L) \mid R \vee S=L\} .
$$

In case $S$ is complemented, the pseudodifference $L \backslash S$ is the complement and in that case we simply refer to it as $S^{\mathrm{C}}$.

For any $a \in L$, the sets

$$
\mathfrak{c}(a)=\uparrow a \quad \text { and } \quad \mathfrak{o}(a)=\{a \rightarrow x \mid x \in L\}=\{x \in L \mid a \rightarrow x=x\}
$$


are special sublocales of $L$ referred to, respectively as closed and open sublocales. They are complements of each other in $\mathcal{S}(L)$. Furthermore, the map $a \mapsto \mathfrak{D}(a)$ is a lattice embedding $L \hookrightarrow \mathcal{S}(L)$ that preserves arbitrary joins. Therefore, denoting by $\mathfrak{b}[L]$ the sublattice of $\mathcal{S}(L)$ consisting of all open sublocales, $L$ and $\mathfrak{o}[L]$ are isomorphic frames.

On the other hand,

$$
a \leq b \text { iff } \mathfrak{c}(a) \supseteq \mathfrak{c}(b), \quad \mathfrak{c}(a) \vee \mathfrak{c}(b)=\mathfrak{c}(a \wedge b) \text { and } \bigcap_{i \in I} \mathfrak{c}\left(a_{i}\right)=\mathfrak{c}\left(\bigvee_{i \in I} a_{i}\right) .
$$

In particular, this means that the set $c[L]$ of closed sublocales of $L$ is a sub-coframe of $\mathcal{S}(L)$.

In the following, given any $A \subseteq L$, we shall denote by $\mathfrak{v}[A]$ and $\mathfrak{c}[A]$ the sets

$$
\mathfrak{v}[A]=\{\mathfrak{v}(a) \mid a \in A\} \quad \text { and } \quad \mathfrak{c}[A]=\{\mathfrak{c}(a) \mid a \in A\} .
$$

Given a sublocale $T$ of $L$, its closure is defined by $\bar{T}=\bigcap\{\mathfrak{c}(a) \mid T \subseteq \mathfrak{c}(a)\}=$ $\mathfrak{c}(\bigwedge T)$. In particular, $\overline{\mathrm{D}(a)}=\mathfrak{c}\left(a^{*}\right)$.

A sublocale $T$ is dense whenever $\bar{T}=L$, that is, $0=\wedge T \in T$. An important distinctive feature of pointfree topology, known as the Isbell's Density Theorem [28], is the existence in any frame of a least dense sublocale, namely its Booleanization

$$
\mathfrak{B}_{L}=\{x \rightarrow 0 \mid x \in L\} .
$$

For any sublocale $T$ of $L, \mathcal{S}(T) \subseteq \mathcal{S}(L)$ (note that, furthermore, if $S$ and $T$ are sublocales of $L$ with $S \subseteq T$, then $S \in \mathcal{S}(T)$ ). Non-empty infima in $\mathcal{S}(T)$, being given by intersection, coincide with infima in $\mathcal{S}(L)$. On the other hand, formula (2.3) shows that arbitrary suprema in $\mathcal{S}(T)$ also coincide with suprema in $\mathcal{S}(L)$ : for any $A_{i} \in \mathcal{S}(T), i \in I$, we have

$$
\bigvee_{i \in I}^{\mathcal{S}(L)} A_{i}=\left\{\bigwedge R \mid R \subseteq \bigcup_{i \in I} A_{i}\right\} \subseteq T
$$

and thus

$$
\bigvee_{i \in I}^{\mathcal{S}(L)} A_{i}=\bigvee_{i \in I}^{\mathcal{S}(T)} A_{i}
$$

Given a sublocale $T$ of $L$ let $\rho_{T}: L \rightarrow T$ be the surjective frame homomorphism defined by

$$
\rho_{T}(a)=\bigwedge\{t \in T \mid t \geq a\} \in T .
$$

It is easy to verify that the open sublocales of $T$ (that we denote as $\mathfrak{v}_{T}(t)$, $t \in T)$ are precisely the intersections $\mathfrak{v}(a) \cap T(a \in L)$ : indeed, $\mathfrak{v}_{T}(t)=\mathfrak{v}(t) \cap T$ for any $t \in T$, and for any $a \in L, \mathfrak{v}(a) \cap T=\mathfrak{v}_{T}\left(\rho_{T}(a)\right)$. Analogously for the closed sublocales. For each $A \subseteq T$ we shall denote by $\mathfrak{D}_{T}[A]$ and $\mathfrak{c}_{T}[A]$ the sets

$$
\mathfrak{v}_{T}[A]=\left\{\mathfrak{b}_{T}(a) \mid a \in A\right\} \quad \text { and } \quad \mathfrak{c}_{T}[A]=\left\{\mathfrak{c}_{T}(a) \mid a \in A\right\} .
$$




\section{Complete eXtremal disconnectedness}

Complete normality formulated in frames appeared for the first time in the literature with [19] (see also [31]), in the following form: a frame is completely normal if every pair $S, T$ of separated sublocales of $L$ (i.e. such that $S \cap \bar{T}=\{1\}=\bar{S} \cap T)$ is separated by open sublocales, that is, there exist open sublocales $U$ and $V$ of $L$ such that $U \cap V=\{1\}, S \subseteq U$ and $T \subseteq V$. As proved in [11, Proposition 3.3], this is equivalent to condition (CN) above. In what follows we will show a dual characterization for condition (CED).

We start with the following easy characterization:

Proposition 3.1. A frame $L$ is completely extremally disconnected if and only if it satisfies the strong De Morgan's law

$$
(a \rightarrow b) \vee(b \rightarrow a)=1
$$

for all $a, b \in L$.

Proof. Sufficiency is obvious since $a \wedge(a \rightarrow b)=a \wedge b$ for any $a, b \in L$. Regarding necessity, let $a, b \in L$. By hypothesis, there are $u, v \in L$ such that $u \vee v=1, a \wedge u \leq b$ and $b \wedge v \leq a$. Hence $1=u \vee v \leq(a \rightarrow b) \vee(b \rightarrow a)$ by adjunction (2.2).

Remark 3.2. Strong De Morgan's law is treated in [21] in the context of toposes. It follows from a result there that this identity holds in $\Omega(X)$ if and only if every closed subspace of $X$ is extremally disconnected ([27]).

The next characterization is the pointfree counterpart of a characterization of hereditary ED presented in [3, Proposition 2.1]. In order to prove it, we need first some technical observations concerning Booleanizations of closed sublocales.

First, it should be remarked that any sublocale $S$ of a frame $L$ is a frame itself with the same meets as in $L$, and since the Heyting operation $\rightarrow$ depends on the meet structure only, with the same Heyting operation. Consequently, for each $a \in L$, the Booleanization of $\mathfrak{c}(a)$ is given by

$$
\mathfrak{B}_{\mathfrak{c}(a)}=\{x \rightarrow a \mid x \in \mathfrak{c}(a)\}=\{x \rightarrow a \mid x \in L\} .
$$

Note that this is the smallest sublocale of $L$ containing $a$. Hence $\mathfrak{B}_{\mathfrak{c}(a)}=\mathfrak{B}_{\mathfrak{c}(b)}$ if and only if $a=b$ (cf. [20, 2.1]).

Lemma 3.3. For any $a, b \in L$ we have:

(a) $\mathfrak{B}_{\mathfrak{c}(a \rightarrow b)}=\mathfrak{v}(a) \cap \mathfrak{B}_{\mathfrak{c}(b)}$.

(b) $\mathfrak{c}(a) \cap \mathfrak{B}_{\mathfrak{c}(b)}=\{1\}$ iff $a \rightarrow b=b$ iff $b \in \mathfrak{v}(a)$.

(c) $\mathfrak{c}(a \rightarrow b)=\overline{\mathfrak{p}(a) \cap \mathfrak{c}(b)}$.

Proof. (a) If $y=x \rightarrow(a \rightarrow b) \in \mathfrak{B}_{\mathfrak{c}(a \rightarrow b)}$ then, using some basic properties of the Heyting operator, we may conclude that

$$
y=a \rightarrow(x \rightarrow b) \in \mathfrak{D}(a) \text { and } y=(x \wedge a) \rightarrow b \in \mathfrak{B}_{\mathfrak{c}(b)} ;
$$


conversely, if $y \in \mathfrak{D}(a) \cap \mathfrak{B}_{\mathfrak{c}(b)}$ then $y=a \rightarrow y=x \rightarrow b$ for some $x \in L$, hence

$$
y=a \rightarrow(x \rightarrow b)=x \rightarrow(a \rightarrow b) \in \mathfrak{B}_{\mathrm{c}(a \rightarrow b)} .
$$

(b) It follows immediately from (1) since $\mathfrak{c}(a) \cap \mathfrak{B}_{\mathfrak{c}(b)}=\{1\}$ iff $\mathfrak{B}_{\mathfrak{c}(b)} \subseteq \mathfrak{v}(a)$ iff $\mathfrak{B}_{\mathfrak{c}(a \rightarrow b)}=\mathfrak{B}_{\mathfrak{c}(b)}$ iff $a \rightarrow b=b$ iff $b \in \mathfrak{v}(a)$.

(c) From $a \rightarrow b \in \mathfrak{v}(a) \cap \mathfrak{c}(b)$ it follows that $\mathfrak{c}(a \rightarrow b) \subseteq \overline{\mathfrak{v}(a) \cap \mathfrak{c}(b)}$. On the other hand, $\mathfrak{c}(b) \subseteq \mathfrak{c}(a \wedge b)=\mathfrak{c}(a) \vee \mathfrak{c}(a \rightarrow b)$ and thus $\mathfrak{v}(a) \cap \mathfrak{c}(b) \subseteq \mathfrak{c}(a \rightarrow b)$. Hence $\overline{\mathfrak{v}(a) \cap \mathfrak{c}(b)} \subseteq \mathfrak{c}(a \rightarrow b)$.

Proposition 3.4. The following are equivalent for a frame $L$ :

(1) $L$ is completely extremally disconnected.

(2) For any open sublocales $A, B$ of $L, \overline{A \cap B^{\mathrm{c}}} \cap \overline{B \cap A^{\mathrm{c}}}=\{1\}$.

(3) For any open sublocales $A, B$ of $L$, there exist open sublocales $U, V$ such that $U \vee V=L, A \cap U \subseteq B$ and $B \cap V \subseteq A$.

Proof. (1) $\Rightarrow(2)$ : Let $A=\mathfrak{v}(a)$ and $B=\mathfrak{v}(b)$ be open sublocales. Then it follows from Lemma $3.3(\mathrm{c})$ and (SDM) that

$$
\overline{A \cap B^{\mathrm{c}}} \cap \overline{B \cap A^{\mathrm{c}}}=\mathfrak{c}(a \rightarrow b) \cap \mathfrak{c}(b \rightarrow a)=\mathfrak{c}((a \rightarrow b) \vee(b \rightarrow a))=\{1\} .
$$

$(2) \Rightarrow(3)$ : Let $A=\mathfrak{v}(a)$ and $B=\mathfrak{v}(b)$ and consider $U=\mathfrak{v}(a \rightarrow b)$ and $V=$ $\mathfrak{v}(b \rightarrow a)$. By hypothesis (and Lemma $3.3(\mathrm{c})$ ) we conclude that

$$
\{1\}=\overline{A \cap B^{\mathrm{c}}} \cap \overline{B \cap A^{\mathrm{c}}}=\mathfrak{c}(a \rightarrow b) \cap \mathfrak{c}(b \rightarrow a)
$$

and thus $U \vee V=\mathfrak{v}(a \rightarrow b) \vee \mathfrak{p}(b \rightarrow a)=L$. On the other hand, $A \cap U=$ $\mathfrak{v}(a) \cap \mathfrak{v}(a \rightarrow b)=\mathfrak{v}(a \wedge b) \subseteq B$. Dually, we have that $B \cap V \subseteq A$.

(3) $\Rightarrow(1)$ : Let $a, b \in L$. By hypothesis there exist open sublocales $U=\mathfrak{o}(u)$ and $V=\mathfrak{v}(v)$ such that $U \vee V=L, \mathfrak{p}(a) \cap U \subseteq \mathfrak{p}(b)$ and $\mathfrak{p}(b) \cap V \subseteq \mathfrak{v}(a)$. Then $u, v \in L$ satisfy (CED).

Now, recall that a sublocale $S$ of $L$ is nowhere dense in $L$ if $S \cap \mathfrak{B}_{L}=\{1\}$ ([20]). We say that two sublocales $S$ and $T$ of $L$ are almost disjoint if $S \cap T$ is a nowhere dense sublocale in both sublocales $S$ and $T$, i.e. $S \cap T \cap \mathfrak{B}_{S}=\{1\}=S \cap T \cap \mathfrak{B}_{T}$.

Next characterization is the pointfree extension of a characterization of completely ED spaces from [4].

Proposition 3.5. The following are equivalent for a frame $L$ :

(1) L is completely extremally disconnected.

(2) Any two almost disjoint closed sublocales of $L$ are disjoint.

(3) Any two separated sublocales of L have disjoint closures.

Proof. $(1) \Rightarrow(2)$ : Let $\mathfrak{c}(a), \mathfrak{c}(b)$ be a pair of almost disjoint closed sublocales. This means that $\mathfrak{c}(b) \cap \mathfrak{B}_{\mathfrak{c}(a)}=\mathfrak{c}(a) \cap \mathfrak{c}(b) \cap \mathfrak{B}_{\mathfrak{c}(a)}=\{1\}$ and $\mathfrak{c}(a) \cap \mathfrak{B}_{\mathfrak{c}(b)}=$ $\mathfrak{c}(a) \cap \mathfrak{c}(b) \cap \mathfrak{B}_{\mathfrak{c}(b)}=\{1\}$. Therefore, by Lemma 3.3 (b), $a \rightarrow b=b$ and $b \rightarrow a=a$. Finally, by (SDM) we conclude that $a \vee b=1$, that is, $\mathfrak{c}(a) \cap \mathfrak{c}(b)=\{1\}$.

$(2) \Rightarrow(3)$ : Let $S, T$ be a pair of separated sublocales and $\bar{S}=\mathfrak{c}(a)$ and $\bar{T}=\mathfrak{c}(b)$. 
Then $\bar{S} \cap T=\{1\}$, that is, $T \subseteq \mathfrak{v}(a)$. Moreover, since $b=\bigwedge T \in T \subseteq \mathfrak{v}(a)$, it follows from Lemma 3.3 (b) that

$$
\bar{S} \cap \bar{T} \cap \mathfrak{B}_{\bar{T}} \subseteq \bar{S} \cap \mathfrak{B}_{\bar{T}}=\mathfrak{c}(a) \cap \mathfrak{B}_{\mathfrak{c}(b)}=\{1\} .
$$

Analogously, $\bar{S} \cap \bar{T} \cap \mathfrak{B}_{\bar{S}}=\{1\}$. Consequently, $\bar{S}$ and $\bar{T}$ are almost disjoint and hence disjoint, by hypothesis.

(3) $\Rightarrow(1)$ : Let $a, b \in L$ and $S=\mathfrak{v}(a) \cap \mathfrak{c}(b)$ and $T=\mathfrak{v}(b) \cap \mathfrak{c}(a)$. Then $\bar{S} \cap T \subseteq$ $\mathfrak{c}(b) \cap \mathfrak{v}(b)=\{1\}$ and $S \cap \bar{T} \subseteq \mathfrak{v}(a) \cap \mathfrak{c}(a)=\{1\}$, hence $S$ and $T$ are separated. By the hypothesis and Lemma $3.3(\mathrm{c}), \bar{S}=\mathfrak{c}(a \rightarrow b)$ and $\bar{T}=\mathfrak{c}(b \rightarrow a)$ are disjoint, that is, $(a \rightarrow b) \vee(b \rightarrow a)=1$.

\section{Complete eXtremal DisconNeCtedness vs. COMPLETE NORMALity}

The duality expressed in 1.4 may be formulated in a more extended setting permitting a simultaneous treatment of several variants of normality and extremal disconnectedness (as proposed in [17]). In what follows we shall speak about a lattice $L$ being normal (resp. extremally disconnected) with respect to some fixed $A \subseteq L$. In this terminology, $L$ is $A$-normal or completely A-normal ( $A-\mathrm{N}$ or $A-\mathrm{CN}$ for short) if it satisfies condition $(\mathrm{N})$ or (CN) of Definition 1.4 with $a, b, u, v \in A$. Analogously, $L$ is extremally A-disconnected or completely extremally $A$-disconnected ( $A$-ED or $A$-CED for short) if it satisfies condition (ED) or (CED) of Definition 1.4 with $a, b, u, v \in A$.

Examples 4.1. The standard example for $A$ is $L$; then, the $A$-notions are just the standard notions. In the case of a frame $L$, the case in which we are most interested, there are other interesting examples (cf. [17]):

(1) $A=\operatorname{Reg} L$ : in this case, $A$-normal = mildly normal and extremally $A$-disconnected $=$ extremally disconnected.

(Recall that the regular part of $L$, denoted $\operatorname{Reg} L$, is the set of all regular elements of $L$, that is, the elements $a \in L$ such that $a^{* *}=a$.)

(2) $A=\delta$-Reg $L: A$-normal $=\delta$-normal and extremally $A$-disconnected $=$ extremally $\delta$-disconnected.

(An element $a$ of $L$ is $\delta$-regular whenever $a=\bigvee_{n \in \mathbb{N}} a_{n}$ with $a_{n}<a$, i.e., $a_{n}^{*} \vee a=1$, and the set of all $\delta$-regular elements is denoted by $\delta$-Reg L.)

(3) $A=\operatorname{Coz} L$ : in this case, any frame is $A$-normal while the class of extremally $A$-disconnected frames is precisely the class of $F$-frames.

(The cozero part of $L$, denoted $\operatorname{Coz} L$, is the set of all cozero elements of $L$, that is, the elements $a \in L$ such that $a=\bigvee_{n} a_{n}$ for some $a_{n} \ll a$, $n=1,2, \cdots$, where $x \ll a$ expresses the familiar relation that $x$ is really inside, or completely below, $a$. This is the largest interpolative relation contained in $<$.)

However, we cannot apply the dualizing process 1.4 directly in frames since the dual lattice of a frame is not a frame in general. 
Yet, if all elements in $A$ are complemented in $L$ then, for the set $A^{\mathrm{C}}$ of complements of all elements in $A, A^{\mathrm{C}}$-normality is precisely extremal $A$-disconnectedness while extremal $A^{\mathrm{C}}$-disconnectedness is $A$-normality, and the two notions may be treated simultaneously.

Therefore the familiar result that the sublocale lattice $\mathcal{S}(L)$ is isomorphic to the coframe obtained by freely adjoining to $L^{o p}$ a complement for each $a \in L$ provides a way of dealing with the duality in frames ([17]). Indeed, it suffices to embed $L$ in the coframe $\mathcal{S}(L)$ via the isomorphism $L \cong \mathfrak{v}[L] \subseteq \mathcal{S}(L)$. Then $L$ will be (completely) $A$-normal iff the lattice $\mathcal{S}(L)$ is (completely) $\mathfrak{v}[A]$-normal. But now, any element of $\mathfrak{v}[A]$ is complemented, hence $L$ will be (completely) $A$-normal iff $\mathcal{S}(L)$ is (completely) extremally c $[A]$-disconnected.

Similarly, $L$ will be (completely) extremally $A$-disconnected iff the lattice $\mathcal{S}(L)$ is (completely) extremally $\mathfrak{o}[A]$-disconnected, that is, (completely) $\mathrm{c}[A]$-normal.

To illustrate this idea we have a first result that characterizes completely $A$-normal frames and completely extremally $A$-disconnected frames (for any $A \subseteq L$ ) at once, with a single proof. Before presenting it, we need to introduce some terminology and notation.

For $\mathcal{A}=\mathfrak{v}[A]$ or $\mathcal{A}=\mathfrak{c}[A]$ let $\mathcal{A}^{\mathfrak{c}}$ be the set of complements of all elements of $\mathcal{A}$. We say that two sublocales $S$ and $T$ of $L$ are $\mathcal{A}$-separated if there exist $F, G \in \mathcal{A}^{\mathrm{C}}$ such that

$$
S \subseteq F, T \subseteq G \text { and } S \cap G=\{1\}=T \cap F .
$$

Further, we say that $S$ and $T$ are separated by $\mathcal{A}$-sublocales if there exist sublocales $U$ and $V$ in $\mathcal{A}$ such that

$$
U \cap V=\{1\}, S \subseteq U \text { and } T \subseteq V .
$$

Remark 4.2. We may speak further about the $A$-closure of a sublocale $T$, that is, $\mathrm{cl}_{A}(T)=\bigcap\{\mathfrak{c}(a) \mid T \subseteq \mathfrak{c}(a), a \in A\}$ (for $A=L$ this is just the standard closure). Whenever $A$ is closed under joins, $\mathrm{cl}_{A}(T)$ belongs to $\mathrm{c}[A]$ and it is of course the smallest sublocale in $c[A]$ that contains $T$. Then it is easy to check that sublocales $S$ and $T$ are $\mathfrak{o}[A]$-separated if and only if $S \cap \mathrm{cl}_{A}(T)=\{1\}=\mathrm{cl}_{A}(S) \cap T$. The selection $A=L$ recovers this way the original definition in [19].

Proposition 4.3. For any frame $L$ and any $A \subseteq L$, the following are equivalent for $\mathcal{A}=\mathfrak{v}[A]$ or $\mathcal{A}=\mathfrak{c}[A]:$

(1) $\mathcal{S}(L)$ is completely $\mathcal{A}$-normal.

(2) Every pair of $\mathcal{A}$-separated sublocales of $L$ is separated by $\mathcal{A}$-sublocales.

Proof. (1) $\Rightarrow(2)$ : Let $S$ and $T$ be $\mathcal{A}$-separated. Then there exist $F, G \in \mathcal{A}^{C}$ such that $S \subseteq F, T \subseteq G$ and $S \cap G=\{1\}=T \cap F$. Of course, $F^{\mathrm{C}}, G^{\mathrm{C}} \in \mathcal{A}$ and therefore, by hypothesis, there exist $U, V \in \mathcal{A}$ such that $U \cap V=\{1\}$, $G^{\mathrm{C}} \subseteq F^{\mathrm{C}} \vee U$ and $F^{\mathrm{C}} \subseteq G^{\mathrm{C}} \vee V$. Moreover, $\{1\}=S \cap G \supseteq S \cap F \cap U^{\mathrm{C}}=S \cap U^{\mathrm{c}}$, from which it follows that $S \subseteq U$, and $\{1\}=T \cap F \supseteq T \cap G \cap V^{\mathrm{C}}=T \cap V^{\mathrm{c}}$, from which it follows that $T \subseteq V$. 
(2) $\Rightarrow(1)$ : Let $F, G \in \mathcal{A}$ and $S=F^{\mathrm{C}} \cap G, T=F \cap G^{\mathrm{c}}$. Clearly, $S \subseteq F^{\mathrm{c}}, T \subseteq G^{\mathrm{c}}$ and $S \cap G^{\mathrm{C}}=\{1\}=F \cap T^{\mathrm{C}}$. By hypothesis, there exist $U, V \in \mathcal{A}$ satisfying $U \cap V=\{1\}, S \subseteq U$ and $T \subseteq V$. This means that

$$
\begin{aligned}
& F^{\mathrm{C}} \cap G=S \subseteq U \Leftrightarrow F^{\mathrm{C}} \cap G \cap U^{\mathrm{c}}=\{1\} \Leftrightarrow G \subseteq F \vee U \\
& F \cap G^{\mathrm{c}}=T \subseteq V \Leftrightarrow F \cap G^{\mathrm{c}} \cap V^{\mathrm{c}}=\{1\} \Leftrightarrow F \subseteq G \vee V .
\end{aligned}
$$

Corollary 4.4. For any frame $L$ and any $A \subseteq L$, the following are equivalent:

(1) L is completely A-normal.

(2) Every pair of $\mathfrak{v}[A]$-separated sublocales of $L$ is separated by $\mathfrak{v}[A]$-sublocales.

Corollary 4.5. For any frame $L$ and any $A \subseteq L$, the following are equivalent:

(1) $L$ is completely A-disconnected.

(2) Every pair of $\mathrm{c}[A]$-separated sublocales of $L$ is separated by $\mathrm{c}[A]$-sublocales.

Proposition 4.3 can be expanded to the next result which extends Proposition 3.3 of [11].

Proposition 4.6. For any frame $L$ and any $A \subseteq L$, the following are equivalent for $\mathcal{A}=\mathfrak{v}[A]$ or $\mathcal{A}=\mathfrak{c}[A]:$

(1) $\mathcal{S}(L)$ is completely $\mathcal{A}$-normal.

(2) For every $S, T \in \mathcal{S}(L)$ satisfying $S \subseteq F \subseteq T$ and $S \subseteq G^{c} \subseteq T$ for some $F, G \in \mathcal{A}$, there exist $U, V \in \mathcal{A}$ such that $S \subseteq V \subseteq U^{\mathrm{C}} \subseteq T$.

(3) For every $S, T \in \mathcal{S}(L)$ satisfying $S \vee F=L=G \vee T$ for some $F, G \in \mathcal{A}$ such that $F \subseteq T$ and $G \subseteq S$, there exist $U, V \in \mathcal{A}$ such that $U \cap V=\{1\}, V^{\mathrm{C}} \subseteq S$ and $U^{\mathrm{c}} \subseteq T$.

Proof. (1) $\Rightarrow$ (2): Let $S \subseteq F \subseteq T$ and $S \subseteq G^{C} \subseteq T$. By (1), there are $U, V \in \mathcal{A}$ such that $U \cap V=\{1\}, G \subseteq F \vee U$ and $F \subseteq G \vee V$. Of course, $V \subseteq U^{c}$. Moreover,

$$
\begin{aligned}
& S \subseteq F \cap G^{\mathrm{c}} \subseteq(G \vee V) \cap G^{\mathrm{c}}=V \cap G^{\mathrm{c}} \subseteq V \quad \text { and } \\
& U^{\mathrm{C}}=\left(U^{\mathrm{c}} \cap F\right) \vee\left(U^{\mathrm{c}} \cap F^{\mathrm{c}}\right) \subseteq\left(U^{\mathrm{c}} \cap F\right) \vee G^{\mathrm{c}} \subseteq T .
\end{aligned}
$$

(2) $\Rightarrow$ (3): Let $S \vee F=L=G \vee T$ as in the hypothesis. Then $L \backslash S \subseteq F \subseteq T$ and $L \backslash S \subseteq G^{\mathrm{C}} \subseteq T$. By (2) there exist $U, V \in \mathcal{A}$ such that $L \backslash S \subseteq V \subseteq U^{\mathrm{C}} \subseteq T$. Clearly, $U \cap V=\{1\}, V^{\mathrm{C}} \subseteq L \backslash(L \backslash S) \subseteq S$ and $U^{\mathrm{C}} \subseteq T$.

(3) $\Rightarrow(1)$ : Let $F, G \in \mathcal{A}$ and consider $S=F^{\mathrm{C}} \vee G$ and $T=F \vee G^{\mathrm{C}}$. Clearly, $S \vee F=L=G \vee T, F \subseteq T$ and $G \subseteq S$. Consequently, by (3), there are $U, V \in \mathcal{A}$ such that $U \cap V=\{1\}, V^{\mathrm{C}} \subseteq S$ and $U^{\mathrm{C}} \subseteq T$. Then, finally, $F \vee U \supseteq F \vee T^{\mathrm{c}}=F \vee G \supseteq G$ and $G \vee V \supseteq G \vee S^{\mathrm{c}}=G \vee F \supseteq F$.

Again, the case $\mathcal{A}=\mathfrak{c}[A]$ yields immediately the dual result for ED:

Corollary 4.7. For any frame $L$ and any $A \subseteq L$, the following are equivalent:

(1) L is completely A-disconnected.

(2) For every $S, T \in \mathcal{S}(L)$ satisfying $S \subseteq \mathfrak{c}(a) \subseteq T$ and $S \subseteq \mathfrak{v}(b) \subseteq T$ for some $a, b \in A$, there exist $u, v \in A$ such that $S \subseteq \mathfrak{c}(v) \subseteq \mathfrak{v}(u) \subseteq T$. 
(3) For every $S, T \in \mathcal{S}(L)$ satisfying $S \vee \mathfrak{c}(a)=L=\mathfrak{c}(b) \vee T$ for some $a, b \in A$ such that $\mathfrak{c}(a) \subseteq T$ and $\mathfrak{c}(b) \subseteq S$, there exist $u, v \in A$ such that $u \vee v=1, \mathfrak{v}(v) \subseteq S$ and $\mathrm{p}(u) \subseteq T$.

\section{Heredity properties}

Recall from Section 1 that an $L=\Omega(X)$ is hereditary ED if and only it is completely ED. In this section we prove that this equivalence extends to arbitrary frames by proving the equivalence between completely $A$-normal frames and hereditarily $A$-normal frames (for any sublattice $A$ of $L$ ).

A frame $L$ is called hereditarily normal [14] whenever every sublocale of $L$ is normal. Dually, we say that $L$ is called hereditarily ED if every sublocale of $L$ is ED.

More generally, for a fixed $A \subseteq L$, we say that $L$ is hereditarily $A$-normal in case every sublocale $T$ of $L$ is $A_{T}$-normal, for $A_{T}=\rho_{T}[A] \subseteq T$ (recall the mapping $\rho_{T}$ ). This is the same as saying that the lattice $\mathcal{S}(T)$ is $\mathfrak{D}_{T}\left[A_{T}\right]$-normal for every sublocale $T$ of $L$. Dually, we say that $L$ is hereditarily $A$-disconnected if every sublocale $T$ of $L$ is $A_{T}$-disconnected, that is, $\mathcal{S}(T)$ is $\mathfrak{c}_{T}\left[A_{T}\right]$-normal for every sublocale $T$ of $L$.

For any sublocale $T$ of $L$ and $\mathcal{A} \subseteq \mathcal{S}(L)$ let $\mathcal{A}_{T}=\{S \cap T \mid S \in \mathcal{A}\} \subseteq \mathcal{S}(T)$. Then, for the case of a sublattice $A \subseteq L$ we have:

Theorem 5.1. For any frame $L$ and any sublattice $A$ of $L$, the following are equivalent for $\mathcal{A}=\mathfrak{v}[A]$ or $\mathcal{A}=\mathfrak{c}[A]$ :

(1) $\mathcal{S}(L)$ is completely $\mathcal{A}$-normal.

(2) For each $T \in \mathcal{S}(L), \mathcal{S}(T)$ is $\mathcal{A}_{T}$-normal.

(3) For each $T \in \mathcal{A}, \mathcal{S}(T)$ is $\mathcal{A}_{T}$-normal.

Proof. (1) $\Rightarrow(2)$ : Let $T$ be a sublocale of $L$ and $F_{T}=F \cap T, G_{T}=G \cap T$ (with $F, G \in \mathcal{A})$ such that $F_{T} \vee G_{T}=T$, that is, $F \vee G \supseteq T$. By hypothesis there exist $U, V \in \mathcal{A}$ such that $U \cap V=\{1\}, F \vee U \supseteq G$ and $G \vee V \supseteq F$. Then $U_{T}=U \cap T, V_{T}=V \cap T \in \mathcal{A}_{T}$ and $U_{T} \cap V_{T}=\{1\}$. On the other hand, by (2.4),

$$
F_{T} \vee U_{T}=(F \vee U) \cap T \supseteq F_{T} \vee G_{T}=T
$$

and, similarly, $G_{T} \vee V_{T}=T$. This shows that $\mathcal{S}(T)$ is $\mathcal{A}_{T}$-normal.

$(2) \Rightarrow(3)$ : It is obvious.

$(3) \Rightarrow(1)$ : We shall prove that $\mathcal{S}(L)$ is completely $\mathcal{A}$-normal using Proposition 4.3. So let $S$ and $T$ be $\mathcal{A}$-separated and $F, G \in \mathcal{A}^{c}$ such that $S \subseteq F, T \subseteq G$ and $S \cap G=\{1\}=F \cap T$ and consider

$$
R=F^{\mathrm{C}} \vee G^{\mathrm{C}} \in \mathcal{A}
$$

( $R$ is indeed in $\mathcal{A}$ since $A$ being a sublattice of $L$ means that both $\mathfrak{v}[A]$ and $\mathfrak{c}[A]$ are closed under finite joins). By hypothesis, $\mathcal{S}(R)$ is $\mathcal{A}_{R}$-normal. Since $F^{\mathrm{C}}=F^{\mathrm{C}} \cap R, G^{\mathrm{C}}=G^{\mathrm{C}} \cap R \in \mathcal{A}_{R}$ and $F^{\mathrm{C}} \vee G^{\mathrm{C}}=R$, there exist $U, V \in \mathcal{A}$ such that $(U \cap R) \cap(V \cap R)=\{1\}$ and $F^{\mathrm{C}} \vee(U \cap R)=R=G^{\mathrm{C}} \vee(V \cap R)$. 
Since $A$ is a sublattice of $L$ it follows that $\mathrm{v}[A]$ and $\mathrm{c}[A]$ are closed under finite meets and so $U^{\prime}=U \cap G^{\mathrm{C}} \in \mathcal{A}$ and $V^{\prime}=V \cap F^{\mathrm{C}} \in \mathcal{A}$. We have

$$
U^{\prime} \cap V^{\prime}=\left(U \cap G^{\mathrm{C}}\right) \cap\left(V \cap F^{\mathrm{C}}\right) \subseteq U \cap V \cap R=\{1\} .
$$

Moreover, from $F^{\mathrm{C}} \vee(U \cap R)=R$ it follows that $R \subseteq F^{\mathrm{C}} \vee U$. Then $S \cap U^{\mathrm{c}} \subseteq$ $F \cap U^{\mathrm{C}} \subseteq R^{\mathrm{C}}=F \cap G \subseteq G$ and thus $S \cap\left(U^{\mathrm{C}} \vee G\right) \subseteq S \cap G=\{1\}$. Hence $S \subseteq U^{\prime}$. Similarly, $T \subseteq V^{\prime}$.

For $\mathcal{A}=\mathfrak{v}[A]$ we have

$$
\mathcal{A}_{T}=\{\mathfrak{o}(a) \cap T \mid a \in A\}=\left\{\mathfrak{o}_{T}\left(\rho_{T}(a)\right) \mid a \in A\right\}=\mathfrak{o}_{T}\left(A_{T}\right)
$$

and hence we get:

Corollary 5.2. For any frame $L$ and any sublattice $A$ of $L$, the following are equivalent:

(1) L is completely A-normal.

(2) $L$ is hereditarily A-normal.

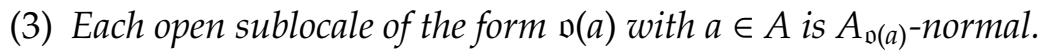

In particular, for $A=L$ we have $A_{\mathfrak{v}(a)}=\mathfrak{v}(a)$ and, therefore, both Theorem 3.7 of [11] and Proposition 3.3 of [14] follow:

Corollary 5.3. The following are equivalent for any frame $L$ :

(1) $L$ is completely normal.

(2) $L$ is hereditarily normal.

(3) Each open sublocale of $L$ is normal.

For $\mathcal{A}=\mathrm{c}[A]$ we have

$$
\mathcal{A}_{T}=\{\mathfrak{c}(a) \cap T \mid a \in A\}=\left\{\mathfrak{c}_{T}\left(\rho_{T}(a)\right) \mid a \in A\right\}=\mathfrak{c}_{T}\left(A_{T}\right)
$$

and hence we also get:

Corollary 5.4. For any frame $L$ and any sublattice $A$ of $L$, the following are equivalent:

(1) $L$ is completely A-disconnected.

(2) $L$ is hereditarily A-disconnected.

(3) Each closed sublocale of the form $\mathfrak{c}(a)$ with $a \in A$ is $A_{\mathfrak{c}(a) \text {-normal. }}$

Finally, for $A=L$ we have $A_{\mathfrak{c}(a)}=\mathfrak{c}(a)$ and we get immediately the announced result for ED:

Corollary 5.5. The following are equivalent for any frame L:

(1) L is completely extremally disconnected.

(2) L is hereditarily extremally disconnected.

(3) Each closed sublocale of $L$ is extremally disconnected.

Remark 5.6. Since a topological space has typically more sublocales than subspaces, one cannot conclude automatically that the pointfree notions 
of hereditary normality and hereditary extremal disconnectedness are conservative extensions of their classical counterparts. Yet this follows now immediately from Corollaries 5.3 and 5.5 and Remark 2.1.

\section{Heredity AND DENSITY}

A property $P$ of frames is said to be hereditary if each sublocale of a frame satisfying $P$ also satisfies $P$, and it is said to be closed-hereditary [resp., open-hereditary] if it is hereditary for closed [resp., open] sublocales.

A natural question arises: when checking hereditary normality of a frame $L$ do we really need to check normality of all the open sublocales? We end the paper with the new observation that it suffices to check it for dense and open sublocales. One of possible arguments for it depends only on the fact that normality is closed-hereditary and its heredity is equivalent to its open-heredity (examples of the same situation include collectionwise normality and $\kappa$-collectionwise normality for frames [16]):

Proposition 6.1. Let $P$ be a property of frames which is closed-hereditary and such that heredity of $P$ is equivalent to open-heredity. For $L$ a frame the following are equivalent:

(1) Each sublocale of L has property P.

(2) Each dense sublocale of $L$ has property $P$.

(3) Each open and dense sublocale of $L$ has property $P$.

Proof. (3) $\Rightarrow(1)$ : This is the only non-trivial implication that needs to be shown. Consider an open sublocale $\mathfrak{p}(a)$ and its closure $\overline{\mathfrak{p}(a)}=\mathfrak{c}\left(a^{*}\right)$. The sublocale

$$
S=\mathfrak{p}(a) \vee\left(L \backslash \mathfrak{c}\left(a^{*}\right)\right)=\mathfrak{v}(a) \vee \mathfrak{p}\left(a^{*}\right)=\mathfrak{p}\left(a \vee a^{*}\right)
$$

is also open. Further, $S$ is dense in $L$ :

$$
\bar{S}=\mathfrak{c}\left(\left(a \vee a^{*}\right)^{*}\right)=\mathfrak{c}\left(a^{*} \wedge a^{* *}\right)=\mathfrak{c}(0)=L .
$$

Thus, $S$ has property $P$. Moreover, $S \cap \mathfrak{c}\left(a^{*}\right)=\mathfrak{p}(a) \cap \mathfrak{c}\left(a^{*}\right) \subseteq \mathfrak{v}(a)$, that is, $\mathfrak{D}(a)=\mathfrak{c}\left(a^{*}\right) \cap S$. Hence $\mathfrak{v}(a)$ is closed in $S$. Since $P$ is closed-hereditary, then $\mathfrak{v}(a)$ has also the property and the conclusion follows from the assumption that heredity of $P$ is equivalent to open-heredity.

Remark 6.2. It is interesting that when $P=$ normality, we can prove the implication $(3) \Rightarrow(1)$ without assuming that heredity of $P$ is equivalent to open-heredity. This is a novelty even for the case of topological normality (see Corollary 6.4). For that, consider an open sublocale $\mathfrak{D}(a)$ of $L$ and $u, v \in \mathfrak{p}(a)$ satisfying

$$
1=u \stackrel{\mathfrak{o}(a)}{\vee} v=a \rightarrow(u \vee v)
$$

By the proof above $S=\mathfrak{v}(a) \vee \mathfrak{v}\left(a^{*}\right)$ is an open and dense sublocale of $L$ hence normal. Moreover, $u, v \in \mathfrak{v}(a) \subseteq S, a \rightarrow(u \vee v)=1$ and $a^{*} \rightarrow(u \vee v)=1$ (since $a^{*}=\bigwedge \mathfrak{p}(a) \leq u \vee v$ ). Hence

$$
u \stackrel{S}{\vee} v=\left(a \vee a^{*}\right) \rightarrow(u \vee v)=(a \rightarrow(u \vee v)) \wedge\left(a^{*} \rightarrow(u \vee v)\right)=1
$$


Then, by the normality of $S$, there exist $x, y \in S$ such that $x \wedge y=0_{S}=0$ and $u \stackrel{S}{\vee} x=1=v \stackrel{S}{\vee} y$. Now consider $a \rightarrow x$ and $a \rightarrow y$ in $\mathfrak{v}(a)$. Notice that

$$
(a \rightarrow x) \wedge(a \rightarrow y)=a \rightarrow(x \wedge y)=a \rightarrow 0=0_{\mathfrak{v}(a)} .
$$

Moreover, $a \leq a \vee a^{*} \leq u \vee x \leq u \vee(a \rightarrow x)$, since $1=u \stackrel{S}{u} x=\left(a \vee a^{*}\right) \rightarrow(u \vee x)$. Hence

$$
u \stackrel{\mathrm{D}(a)}{\vee}(a \rightarrow x)=a \rightarrow(u \vee(a \rightarrow x))=1,
$$

and it can be proved in a similar way that $v \stackrel{\mathfrak{o}(a)}{\vee}(a \rightarrow y)=1$.

After Remark 6.2 we have a small contribution to general topology. The point of the proposition and the corollary which follow should have been known (but is not) since Urysohn told us in his celebrated 1925 paper that it is not necessary to check all subspaces for whether they are normal.

Mimicking the proof in 6.1 for a topological space $X$, an arbitrary open subspace $A$ of $X$, and $S=A \cup(X \backslash \bar{A})$, we get:

Proposition 6.3. Let $P$ be a topological property which is closed-hereditary and such that heredity of $P$ is equivalent to open-heredity. For $X$ a topological space the following are equivalent:

(1) Each subspace of $X$ has property $P$.

(2) Each dense subspace of $X$ has property $P$.

(3) Each open and dense subspace of $X$ has the property $P$.

Corollary 6.4. For X a topological space the following are equivalent:

(1) $X$ is hereditarily normal.

(2) Each dense subspace of $X$ is normal.

(3) Each open and dense subspace of X is normal.

Examples 6.5. Besides topological normality, among examples of topological properties $P$ which are closed-hereditary and such that heredity of $P$ is equivalent to open-heredity of $P$ are the following:

(1) $P=$ collectionwise normality $([10,5.1 . C(a)]$ and $[18$, Lemma 1$])$,

(2) $P=$ compactness $([32$, Theorem 1$])$,

(3) $P=$ Lindelöfness ([10, 3.8.4 and 3.8.A (b)]),

(4) $P=$ paracompactness $([10,5.1 .29$ and 5.1.F (a) $])$.

This list is not exhaustive (cf. e.g. diagram 4.1 in [9]). Another way of extending it is to require cardinal restrictions. As an example, we mention $\mathcal{K}-$ collectionwise normality (cf. $[10,3.8$.A]). We note that hereditarily compact spaces are also called Noetherian spaces. They play an important role in algebraic geometry (cf. [5, Chap. II, § 4, Proposition 9]).

Since normality [resp., extremal disconnectedness] is closed-hereditary [resp., open-hereditary], and extremal disconnectedness is dense-hereditary (cf. [29, Theorem 6.2 (b)]), we have the following enrichment to the 
discussion of heredity in spaces which are both normal and extremally disconnected (cf. [7] and [8]).

Corollary 6.6. For $X$ a topological space the following are equivalent:

(1) Each subspace of $X$ is normal and extremally disconnected.

(2) Each open [closed] subspace of $X$ is normal and extremally disconnected.

(3) Each dense subspace of $X$ is normal and extremally disconnected.

(4) Each open and dense subspace of $X$ is normal and extremally disconnected.

A subset of a topological space is dense in the space if its only closed superset is the whole space. Dually, a subset of a topological space is said to be codense if its only open superset is the whole space. The earliest references for the concept of topological codensity, we are aware of, goes back to [1], [26] and [22].

The concept of codensity trivializes if points are closed. But it has some content otherwise, and gives rise to another interesting result: normality is codense hereditary.

Proposition 6.7. A topological space is normal if and only if each its codense subspace is normal.

Proof. Let $X$ be a normal space and $A$ be its codense subspace. Let $K_{1}$ and $K_{2}$ be closed and disjoint in $A$. Then $K_{1}$ and $K_{2}$ are traces on $A$ of closed subsets $F_{1}$ and $F_{2}$ of $X$. Since $A \subseteq X-\left(F_{1} \cap F_{2}\right)$, hence $F_{1} \cap F_{2}=\varnothing$ on account of $A$ being codense. Hence $F_{1}$ and $F_{2}$ have disjoint open neighborhoods in $X$ whose intersections with $A$ are disjoint open neighborhoods of $K_{1}$ and $K_{2}$ in $A$. Hence $A$ is normal. The converse is obvious, for $X$ is codense in $X$.

We end with the extension of this result to frames. In pointfree topology codense sublocales are considered in [6]: a sublocale $T$ of a frame $L$ is codense if $T^{\circ}=L$, where $T^{\circ}=\bigcap\{\mathfrak{p}(a) \mid T \subseteq \mathfrak{D}(a)\}$ is the fitting closure on sublocales. (Note that a sublocale $T$ is codense if and only if the associated frame surjection $\rho_{T}: L \rightarrow T$ is codense, that is, $\rho_{T}(a)=1$ only if $a=1$ ). Of course, we may now speak about the $\mathcal{A}$-closure of a sublocale $T$ for $\mathcal{A}=\mathfrak{p}[A]$ or $\mathcal{A}=\mathfrak{c}[A]$ (and an arbitrary $A \subseteq L$ ), that is, the sublocale $\mathrm{cl}_{\mathcal{A}}(T)=\bigcap\{A \in \mathcal{A} \mid T \subseteq A\}$ (that may not belong to $\mathcal{A}$, unless $\mathcal{A}$ is closed under intersections). We then say that $S$ is $\mathcal{A}$-dense whenever $\mathrm{cl}_{\mathcal{A}}(T)=L$. The case when $\mathcal{A}=\mathfrak{c}[A]$, with $A=L$, covers the usual density while $\mathcal{A}=\mathfrak{v}[A]$ and $A=L$ covers codensity.

Proposition 6.8. For any frame $L$ and any sublattice $A$ of $L$, the following are equivalent for $\mathcal{A}=\mathfrak{v}[A]$ or $\mathcal{A}=\mathfrak{c}[A]$ :

(1) $\mathcal{S}(L)$ is $\mathcal{A}$-normal.

(2) For each $\mathcal{A}$-dense $T \in \mathcal{S}(L), \mathcal{S}(T)$ is $\mathcal{A}_{T}$-normal.

Proof. The implication (2) $\Rightarrow(1)$ is trivial since $L$ is $\mathcal{A}$-dense. Conversely, let $T$ be an $\mathcal{A}$-dense sublocale of $L$. Consider $F_{1}=G_{1} \cap T$ and $F_{2}=G_{2} \cap T$ in $\mathcal{A}_{T}$ with $F_{1} \vee F_{2}=T$, that is, $T \subseteq G_{1} \vee G_{2} \in \mathcal{A}$ (since $A$ is a sublattice 
of $L$ ) and $G_{1} \vee G_{2}=L$ (since $T$ is $\mathcal{A}$-dense). Hence, by hypothesis, there exist $U_{1}, U_{2} \in \mathcal{A}$ such that $U_{1} \cap U_{2}=\{1\}$ and $G_{1} \vee U_{1}=L=G_{2} \vee U_{2}$. Let $V_{1}=U_{1} \cap T, V_{2}=U_{2} \cap T \in \mathcal{A}_{T}$. Clearly, $V_{1} \cap V_{2}=\{1\}$ and $F_{1} \vee V_{1}=T=$ $F_{2} \vee V_{2}$.

For $\mathcal{A}=\mathfrak{v}[L]$ and $\mathcal{A}=\mathfrak{c}[L]$ we get, respectively:

Corollary 6.9. (1) A frame $L$ is normal iff each codense sublocale of $L$ is normal.

(2) A frame $L$ is extremally disconnected iff each dense sublocale of $L$ is extremally disconnected.

\section{ACKNOWLEDGEMENTS}

We are most grateful to the referee for all valuable comments and suggestions that have helped improve the presentation of the paper.

The research work that originated this paper was started during a visit of JGG and JP to TK in Poznań. The hospitality of Adam Mickiewicz University is gratefully acknowledged. Financial assistance from the Ministry of Economy and Competitiveness of Spain (grant MTM2015-63608-P) and from the Basque Government (grant IT974-16) is also acknowledged. JP also acknowledges support from the Centre for Mathematics of the University of Coimbra (funded by the Portuguese Government through FCT/MEC and co-funded by the European Regional Development Fund through the Partnership Agreement PT2020).

\section{REFERENCES}

[1] B. Banaschewski and R. Harting, Lattice aspects of radical ideals and choice principles, Proc. London Math. Soc. 50 (1985) 385-404.

[2] B. Banaschewski and A. Pultr, On weak lattice and frame homomorphisms, Algebra Universalis 51 (2004) 137-151.

[3] G. Bezhanishvili, N. Bezhanishvili, J. Lucero-Bryan and J. Van Mill, S4.3 and hereditarily extremally disconnected spaces, Georgian Math. J. 22 (2015) 469-475.

[4] A. Blaszczyk, M. Rajagopalan and A. Szymanski, Spaces which are hereditarily extremely disconnected, J. Ramanujan Math. Soc. 8 (1993) 81-94.

[5] N. Bourbaki, Commutative Algebra, Chapter 1-7, Springer-Verlag, Berlin, 1989.

[6] M. M. Clementino, J. Picado and A. Pultr, The other closure and complete sublocales, Appl. Categ. Structures 26 (2018) 891-908.

[7] C. Costantini and A. Marcone, Extensions of functions which preserve the continuity on the original domain, Topology Appl. 103 (2000) 131-153.

[8] C. Costantini and D. Shakhmatov, Examples concerning extensions of continuous functions, Topology Appl. 143 (2004) 189-208.

[9] D. K. Burke, Covering properties, in: K. Kunen and J. E. Vaughan (Eds.), Handbook of Set-Theoretic Topology, Elsevier, Amsterdam, 1984, pp. 347-422.

[10] R. Engelking, General Topology, Polish Sci. Publishers, Warszawa, 1977.

[11] M. J. Ferreira, J. Gutiérrez García and J. Picado, Completely normal frames and real valued functions, Topology Appl. 156 (2009) 2932-2941.

[12] M. J. Ferreira, J. Picado and S. Pinto, Remainders in pointfree topology, Topology Appl. 245 (2018) 21-45.

[13] L. Gillman and M. Jerison, Rings of Continuous Functions, Springer, New YorkHeidelberg, 1976. 
[14] J. Gutiérrez García, T. Kubiak and J. Picado, Monotone insertion and monotone extension of frame homomorphisms, J. Pure Appl. Algebra 212 (2008) 955-968.

[15] J. Gutiérrez García, T. Kubiak and J. Picado, Lower and upper regularizations of frame semicontinuous real functions, Algebra Universalis 60 (2009) 169-184.

[16] J. Gutiérrez García, I. Mozo Carollo, J. Picado and J. Walters-Wayland, Hedgehog frames and a cardinal extension of normality, J. Pure Appl. Algebra, to appear (doi: 10.1016/j.jpaa.2018.08.001).

[17] J. Gutiérrez García and J. Picado, On the parallel between normality and extremal disconnectedness, J. Pure Appl. Algebra 218 (2014) 784-803.

[18] R. E. Hodel, Total normality and the hereditary property, Proc. Amer. Math. Soc. 17 (1966) $462-465$.

[19] J. Isbell, Graduation and dimension in locales, in: Aspects of Topology, London Math. Soc. Lecture Notes 93, pp. 195-210 (1985).

[20] J. Isbell, First steps in descriptive theory of locales, Trans. Amer. Math. Soc. 327 (1991) 353-371.

[21] P. T. Johnstone, Conditions related to De Morgan's law, in: Applications of Sheaves, Lecture Notes in Math. 753, Springer, Berlin (1980), pp. 479-491.

[22] T. Kubiak, Normality versus extremal disconnectedness (unpublished manuscript, 1989).

[23] T. Kubiak, On extremally disconnected subspaces, Fasc. Math. 19 (1990) 143-145.

[24] T. Kubiak, The topological modification of the L-fuzzy unit interval, in: S. E. Rodabaugh, E. P. Klement, U. Höhle (Eds.), Applications of Category Theory to Fuzzy Subsets, Kluwer, Dordrecht, 1992, pp. 276-305, 349.

[25] T. Kubiak and M.A. de Prada Vicente, Hereditary normality plus extremal disconnectedness and insertion of a continuous function, Math. Japon. 46 (1997) 403-405.

[26] S. D. McCartan, Topological equivalents of the axiom of choice, Irish Math. Soc. Bull. 21 (1988) 45-48.

[27] S. B. Niefield and K. I. Rosenthal, Strong De Morgan's law and the spectrum of a commutative ring, J. Algebra 93 (1985) 169-181.

[28] J. Picado and A. Pultr, Frames and locales: Topology without points, Frontiers in Mathematics, vol. 28, Springer, Basel (2012).

[29] J. R. Porter and R.G. Woods, Extensions and Absolutes of Hausdorff Spaces, SpringerVerlag, Berlin, 1988.

[30] H. Simmons, The lattice theoretic part of topological separation properties, Proc. Edinburgh Math. Soc. (2) 21 (1978) 41-48.

[31] B. Šmarda, Completely normal locales, Acta Univ. Carolin. Math. Phys. 31 (1990) 101-104.

[32] A. H. Stone, Hereditarily compact spaces, Amer. J. Math. 82 (1960) 900-914.

[33] W.J. Thron, Lattice-equivalence of topological spaces, Duke Math. J. 29 (1962) 671-679.

Departamento de Matemáticas, Universidad del País Vasco-Euskal Herriko Unibertsitatea, Apartado 644, 48080 Bilbao, SPAIN

Email address: javier.gutierrezgarcia@ehu.eus

Wydziae Matematyki i Informatyki, Uniwersytet im. Adama Mickiewicza, ul. UmulTOWSKa 87, 61-614 Poznań, POLAND

Email address: tkubiak@amu.edu.pl

CMUC, Department of Mathematics, University of Coimbra, Apartado 3008, 3001-501

CoImbra, PORTUGAL

Email address: picado@mat.uc.pt 